Bala, A., \& Gupta, K. (2020). Examining the long memory in stock returns and liquidity in India. Copernican Journal of Finance \& Accounting, 9(3), 25-43. http://dx.doi.org/10.12775/ CJFA.2020.010

\author{
Anju Bala* \\ I.K. Gujral Punjab Technical University \\ KAPIL GUPTA** \\ I.K. Gujral Punjab Technical University

\section{EXAMINING THE LONG MEMORY IN STOCK RETURNS AND LIQUIDITY IN INDIA}

Keywords: emerging market, long memory, persistence and market efficiency.

JEL Classifications: C1, C5, G1, G14.

Abstract: The present study examines the long memory in stock liquidity and returns in Indian equity market by using data for broad indices from January, 1997 to December, 2019 by applying the hurst exponent (1951) rescaled range analysis. It is observed that time varying degree of persistence nature in individual and full series analysis of returns. Moreover, liquidity series exhibit long memory process in Nifty-100, Nifty-200 and Nifty MidCap-50. Findings are consistent with Sadique and Silvapulle (2001), Henry (2002), Cavalcante (2002) and Baum, Barkoulas and Caglayan (1999).

Date of submission: June 25, 2020; date of acceptance: August 18, 2020.

* Contact information: anjubala_attri@yahoo.in, I.K.Gujral Punjab Technical University, Kapurthala-144603, Punjab, India, phone: +91-946-557-4763; ORCID ID: https://orcid.org/0000-0002-5808-8469.

** Contact information: kapilfutures@gmail.com, I.K. Gujral Punjab Technical University, Kapurthala-144603, Punjab, India, phone: +91-947-809-8074; ORCID ID: https:// orcid.org/0000-0003-3817-1772. 


\section{INTRODUCTION}

The presence of long memory supports the notion of market inefficiency in securities market (Onour, 2010). Market inefficiency refers to the fact that it does not immediately absorb the new information flow but it responds gradually and takes a substantial time for relevant information to disseminate across the market (Fama, 1970, 1991) and (Peters, 1994). There is trend to either understate or overstate the effect of such information in stock prices (Barkoulas \& Baum, 1996). If returns series demonstrate the presence of persistence behavior, previous returns can be utilized to estimate the upcoming returns (Hiremath \& Kamaiah, 2010). Therefore, presence of long memory property provides support for framing portfolio strategy, which may also help to generate abnormal profits from the financial investment (Cevik \& Emec, 2013). The investigation on persistence behavior in asset prices, liquidity is essential for the practitioners, since, its presence can have an impact on investment decision, portfolio selection and trading strategies (Balcilar, Ozdemir \& Cakan, 2015; Badhani, 2008).

The seminal research on long memory in capital market was initiated by Hurst (1951). Furthermore, Greene and Fietlitz (1977) and Aydogan and Booth (1988) demonstrated that US stock returns exhibit long memory. However, Lo (1991) did not find significant long memory in US stock returns. Nonetheless, Mandelbrot (1971) confronted that the arbitrage may not be negotiable when long memory is exhibited. Thereafter, Lo (1991) observed that the dynamic behavior in financial markets may be a considerable reason for long memory dynamics.

Furthermore, Hiremath and Kamaiah (2010) and Badhani (2012) found that high volatility, anomalous behavior and market trend are the characteristics of developing economies, which affirms that due to presence of market imperfections, long memory behavior might arise in the developing markets.

Moreover, Badhani $(2006$; 2008) explored the persistence behavior in India and found that stock returns do not report the presence of long memory, whereas absolute returns and squared returns (proxy of volatility) exhibit persistence behavior. On the other hand, subsample covering the duration from March, 2001 to December, 2007 affirms that volatility does not exhibit persistence behavior. 
In addition, Goudarzi (2010) observed persistence behavior in BSE-500 returns, which suggests that BSE 500 returns and volatility are more significant with leverage. Moreover, Ma, Li, Zou and Wu (2006) found significant serial correlation in returns of Chinese stock market. Likewise, Verma (2008) affirms that only three companies out of sixty entail the persistence behavior in returns. Kilic (2004) reported significant serial correlation in the volatility process in Turkey. Furthermore Souza, Tabak and Cajueiro (2008) observed the moderate long memory in European Monetary System using the Hurst Exponent, which suggests that being inside the EMS increases predictability. In addition, Bala and Gupta (2018) reported considerable long-term persistence in Sensex and Nifty returns series. However, volatility series does not contain any persistence behavior but exhibits clustering.

Similarly, Nath and Reddy (2002) examined the persistence behavior in Rupee-Dollar exchange rates and found that there are chances of random walk in three month, while for other time period, it may have mean reverting or persistence tendency. Furthermore, Mahalingam and Selvam (2014) observed high degree of persistence behavior in Indian stock market.

Furthermore, Bhattacharya and Bhattacharya (2012) found persistence behavior in absolute returns along with volatility in international markets. However, evidence did not support the Taylor effect. Moreover, Chen and Diaz (2013) observed significant persistence behavior in green exchange traded funds. Whereas, non-green exchange traded funds did not advocate serial correlation in volatility. Henry (2002) tested the long range dependence in Taiwanese, German and South Korean stock markets and found that persistence behavior is real and not occurred due to shift enhancement and structural breaks of Africa (MENA) vicinity and Middle East in variance. On the contrary, Jayasuriya (2009) advocate that structural changes and persistence behavior in volatility does not show any significant relationship. Similarly, Chung, Lin and Wu (2000) found that Asia-pacific markets hold spurious serial correlation due to shift enhancement in variance series. However, Cevik and Emec (2013) observed persistence behavior in returns series of Turkish stock market.

Moreover, Turkyilmaz and Balibey (2014) examined Pakistan security exchange and found that it is inefficient in weak form and contain serial correlation structure in volatility series also. Cavalcante and Assaf (2004) found significant serial correlation in volatility and return series in Brazilian financial markets. Likewise, Danilenko (2009) found that industrial sector report sig- 
nificant long memory whereas, healthcare and utilities sector entail the weak long range dependence.

In the Indian context, liquidity patterns were studied by Krishnan and Mishra (2013) and Kumar and Misra (2018) for the equity indices and they found that individual stock liquidity co-moves to a high degree with market liquidity and industry liquidity.

Bhattacharya, Sengupta, Bhattacharya and Roychoudhury (2016) found significant degrees of positive association between liquidity and return and noted the order of importance of selected liquidity dimensions in explaining stock market returns.

Cajueiro and Tabak (2008) observed strong long memory in Asian markets than in Latin America. There is considerable support for long memory in stochastic volatility in stock returns. Bhattacharya and Bhattacharya (2012; 2013) and Hull and Mc Groarty (2014) found strong evidence of long memory persistence in volatility over the time. Bariviera (2011) observed that long memory parameter is positively correlated with market capitalization but negatively with an average daily turnover.

In nutshell, a plethora of literature is available in emerging as well as developed markets, however, there is a dearth of empirical research on examining the presence of long memory in India, which is one of the most liquid capital market of the world (Soofi, Wang \& Zhang, 2006), (Krishnan \& Mishra, 2013) and (Goudarzi \& Ramanarayanan, 2011).

The study also tries to pursuit to check whether liquidity and returns both have long memory effect. There is a paucity of literature to study the persistence behavior of Indian Stock Market. Therefore, this paper is an attempt to plug the research gap.

Paper is further organized in four sections. Second section describes the database and research methodology. The results and analysis of the study are discussed in the third section. Discussion and implication of the study presented in fourth section and the conclusion of the study has been presented in the five sections.

\section{RESEARCH METHODOLOGY AND THE COURSES OF THE RESEARCH PROCESS}

This study makes an attempt to study the presence of long-memory in returns and liquidity of Nifty-50, Nifty-100, Nifty-200, Nifty-500, Nifty Next -50, Nifty 
Small Cap 50, Nifty Large MidCap-250, Nifty Full MidCap-100, Nifty Mid Cap150, and Nifty Mid Cap -50 indices using rescale range analysis (hurst exponent). The present study uses daily data from the National Stock Exchange of India (NSE) from January, 1997 to December, 2019 as presented in table 1. The study has also calculated long memory components for each year and full period from January, 1997 to December, 2019 to check whether the presence of long memory is due to structural breaks, regime shift, market friction, political changes and market microstructure etc. that have taken place during the sample period in India.

Table 1. Description of sample size (returns and liquidity)

\begin{tabular}{|l|c|c|c|c|}
\hline \multicolumn{1}{|c|}{ Index } & \multicolumn{1}{|c|}{$\begin{array}{c}\text { No. of } \\
\text { Observations } \\
\text { (N)(Returns) }\end{array}$} & $\begin{array}{c}\text { Sample Period } \\
\text { (Returns) }\end{array}$ & $\begin{array}{c}\text { No. of } \\
\text { Observations } \\
\text { (N) } \\
\text { (Liquidity) }\end{array}$ & $\begin{array}{c}\text { Sample Period } \\
\text { (Liquidity) }\end{array}$ \\
\hline \hline Nifty-50 & 5682 & $1-1-1997$ to 31-12-2019 & 5682 & $1-1-1997$ to 31-12-2019 \\
\hline Nifty-100 & 4323 & $1-1-2003$ to 31-12-2019 & 3636 & 20-9-2005 to 31-12-2019 \\
\hline Nifty-200 & 3972 & $1-1-2004$ to 31-12-2019 & 2090 & 20-7-2011 to 31-12-2019 \\
\hline Nifty-500 & 5219 & $8-6-1999$ to 31-12-2019 & 5219 & $8-6-1999$ to 31-12-2019 \\
\hline Nifty Next -50 & 5721 & $1-1-1997$ to 31-12-2019 & 5721 & $1-1-1997$ to 31-12- 2019 \\
\hline Nifty SmallCap-50 & 3715 & $1-1-2005$ to 31-12-2019 & 923 & $1-4-2016$ to 31-12-2019 \\
\hline Nifty Large MidCap-250 & 3655 & $1-4-2005$ to 31-12-2019 & 681 & 28-3-2017 to 31-12-2019 \\
\hline Nifty Full MidCap-100 & 3547 & $1-4-2005$ to 20-2-2019 & 716 & 4-4-2016 to 20-2-2019 \\
\hline Nifty MidCap-150 & 3651 & $1-4-2005$ to 31-12-2019 & 924 & $1-4-2016$ to 31-12-2019 \\
\hline Nifty Midcap-50 & 3968 & $1-1-2004$ to 31-12-2019 & 3968 & $1-1-2004$ to 31-12-2019 \\
\hline \hline
\end{tabular}

S o u r c e s : compiled by author on the basis of data downloaded from official website of NSE.

\section{Methodology}

Daily returns are computed as the difference in the natural logarithm of the closing index value for the two consecutive trading days. It can be presented is equation 1 :

$$
\mathrm{Rt}=\ln (\mathrm{Pt} / \mathrm{Pt}-1) \text { or } \mathrm{Rt}=\ln (\mathrm{Pt})-\ln (\mathrm{Pt}-1)
$$


Where Rt is natural logarithmic daily return at time t. Pt-1 and Pt are daily prices of stock index at two successive days $\mathrm{t}-1$ and $\mathrm{t}$ respectively.

\section{HURST EXPONENT}

To examine long memory, 'Hurst' exponent is computed. The origin of long memory test can be attributed to Hurst exponent ' $\mathrm{H}$ ', which was developed in 1951 by Hurst to measure water related process. The Hurst exponent (or the self-similarity parameter) is a dimensionless parameter and diverse methodologies exist to estimate it. The concept of Hurst exponent finds its applications in many research fields including the field of financial studies due to the path-breaking works of Mandelbrot, $(1963 ; 1997)$ and Peters $(1994 ; 1996)$. The Hurst exponent lies in the range $0 \leq \mathrm{H} \leq 1$. If the Hurst exponent is 0.5 then the process is said to follow a random walk. When the Hurst exponent is more than 0.5 , it suggests positive long-range autocorrelation or persistence in the series. On the other hand, when the Hurst exponent is smaller than 0.5, it suggests the presence of negative autocorrelation or means reversion in the series (Kumar, 2004), (Chen \& Huang, 2014), (Gayathri, Murugesan \& Gayathri, 2012) and (Kumar, 2014).

Table 2. Hurst exponent coefficient

\begin{tabular}{|l|l|}
\hline \hline \multicolumn{2}{|c|}{ The values of Hurst exponent range between $\mathbf{0}$ and $1:$} \\
\hline \hline $0<\mathrm{H}<0.5$ & Anti-persistence \\
\hline $\mathrm{H}=0.5$ & Random walk \\
\hline $0.5<\mathrm{H}<1$ & Persistence \\
\hline \hline
\end{tabular}

S o u r c e s: presented by author to show the range of hurst exponent (Kumar, 2004).

\section{Hurst Exponent AND Rescaled RANGe (R/S) ANALysis}

Qian and Rasheed (2004) and Cajueiro and Tabak (2005) suggested that the Hurst exponent can be calculated by rescaled range analysis (R/S analysis). For a time series, $\mathrm{X}=\mathrm{X} 1, \mathrm{X} 2, \ldots \mathrm{Xn}, \mathrm{R} / \mathrm{S}$ analysis method is as follows: 
$\mathrm{n}$

(1) Calculate mean value $\mathrm{m} .=1 / \mathrm{n} \sum \mathrm{Xi} \mathrm{i}=1$

(2) Calculate mean adjusted series $Y$ : $Y t=X t-m, t=1,2, \ldots, n t$

(3) Calculate cumulative deviate series $\mathrm{Z}: \sum \mathrm{Yi}, \mathrm{t}=1,2, \ldots, \mathrm{n} \mathrm{i}=1$

(4) Calculate range series $\mathrm{R}$ : $\mathrm{Rt}=\max (\mathrm{Z1}, \mathrm{Z2}, \ldots, \mathrm{Zt})-\min (\mathrm{Z1}, \mathrm{Z} 2, \ldots, \mathrm{Zt}) \mathrm{t}=1$, $2, . ., \mathrm{nt} 2$

(5) Calculate standard deviation series $\mathrm{St}=\sqrt{1} \mathrm{n} \sum(\mathrm{Xi}-\mathrm{u})$ $\mathrm{t}=1,2, \ldots \ldots, \mathrm{n} \mathrm{i}=1$

Here, $\mathrm{u}$ is the mean value from $\mathrm{X} 1$ to $\mathrm{Xt}$.

(6) Calculation of Rescaled Range $\operatorname{Series}(\mathrm{R} / \mathrm{S})$ : $(\mathrm{R} / \mathrm{S}) \mathrm{t}=\mathrm{Rt} / \mathrm{St}$ where, $\mathrm{t}=1$, $2, \ldots, \mathrm{n}$

Note: $(R / S)$ it is averaged over the regions [X1, Xt], $[X t+1, X 2 t]$ until $[X(m-1)$ $\mathrm{t}+1, \mathrm{Xmt}$ ] where $\mathrm{m}=$ floor( $\mathrm{n} / \mathrm{t})$. In practice, to use all data for calculation, a value of $t$ is chosen that is divisible by $n$.

\section{RESULTS AND ANALYSIS}

Before discussing the long memory estimation results through Hurst exponent procedure proposed in third section and comparing the efficiency in reducing the portfolio risk, it is important to discuss the series properties of under examination. Results in table 3 indicate the information relating to the summary of full sample period for returns and liquidity in Nifty Small-Cap 50, Nifty LargeMidCap-250, Nifty MidCap-150, Nifty-50, Nifty-100, Nifty-200, Nifty-500, Nifty Next -50, Nifty Full MidCap-100, and Nifty Mid Cap -50 (Barkoulas, Baum \& Travlos, 2000). Table 3 provides that returns series of all indices reports significantly persistent behavior. The estimated coefficient of $\mathrm{H}$ exponent suggests that all indices exhibit long memory in returns series, which implies that past returns could forecast the upcoming returns (Chow, Denning, Ferris \& Noronha, 1995) and (Baillie, 1996). These findings would be helpful to understand the behavior of the market for an investor, policymakers and portfolio managers to decide where they would get abnormal profits by using long memory insights data Henry (2002), Lillo and Farmer (2004), Garvey and Gallagher (2009), Cavalcante and Assaf (2004). However, finding in liquidity series shows that only Nifty MidCap-50 exhibits long memory component (Ozun \& Cifter, 2007; Barkoulas, Baum \& Travlos, 2000). 


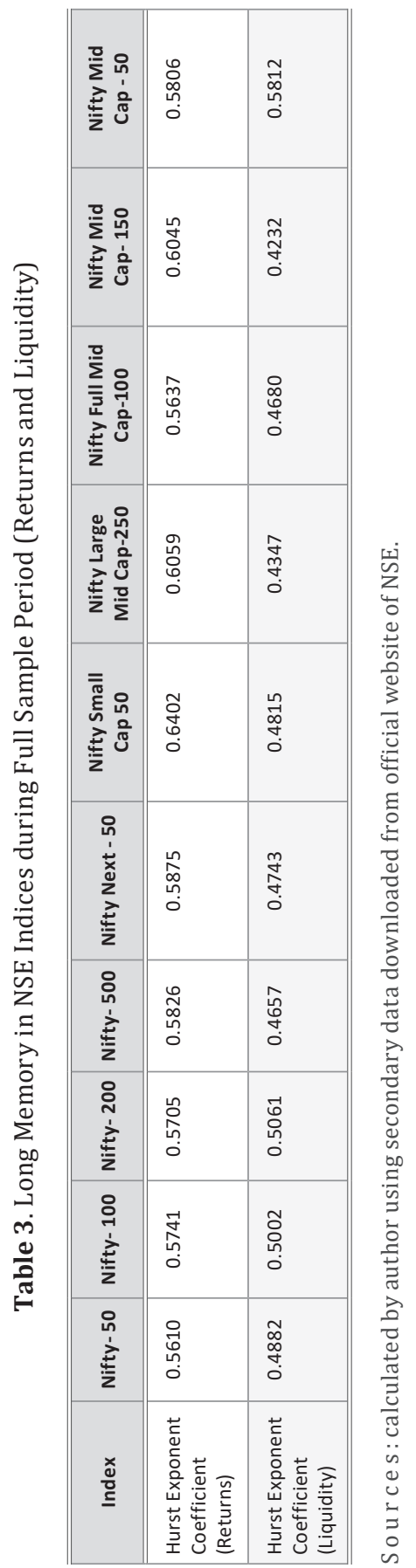




\begin{tabular}{|c|c|c|c|c|c|c|c|c|c|c|c|c|c|c|c|c|c|}
\hline 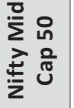 & $\Sigma$ & $\Sigma$ & $\Sigma$ & $\Sigma$ & $\Sigma$ & $\Sigma$ & $\Sigma$ & 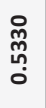 & $\begin{array}{l}\stackrel{\infty}{\tilde{N}} \\
\stackrel{n}{0}\end{array}$ & $\begin{array}{l}\overrightarrow{1} \\
0 \\
0 \\
0 \\
0\end{array}$ & $\begin{array}{l}\text { 足 } \\
\text { م⿱⺈口} \\
0 \\
0\end{array}$ & $\begin{array}{l}\text { 节 } \\
\text { ڤn. } \\
0\end{array}$ & $\begin{array}{l}\tilde{N} \\
0 \\
0 \\
0\end{array}$ & 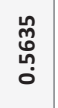 & $\begin{array}{l}0 \\
\infty \\
\Re ̂ n \\
\end{array}$ & $\begin{array}{l}\hat{\tilde{E}} \\
0 \\
0\end{array}$ & $\begin{array}{l}\text { ป̃ } \\
0 \\
0 \\
0\end{array}$ \\
\hline 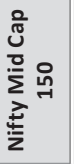 & $\Sigma$ & $\Sigma$ & $\Sigma$ & $\Sigma$ & $\Sigma$ & $\Sigma$ & $\Sigma$ & $\Sigma$ & 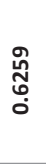 & 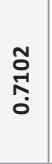 & 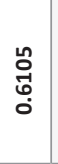 & $\begin{array}{l}\text { 象 } \\
\text { مొ } \\
0 \\
0\end{array}$ & $\begin{array}{l}0 \\
\infty \\
: \\
6 \\
0\end{array}$ & 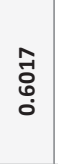 & 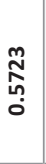 & $\begin{array}{l}\tilde{n} \\
\stackrel{\tilde{E}}{0} \\
\stackrel{0}{0}\end{array}$ & 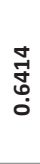 \\
\hline 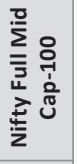 & $\Sigma$ & $\Sigma$ & $\Sigma$ & $\Sigma$ & $\Sigma$ & $\Sigma$ & $\Sigma$ & $\Sigma$ & $\begin{array}{l}-1 \\
00 \\
0 \\
0\end{array}$ & 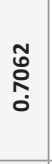 & 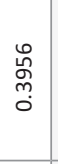 & $\begin{array}{l}\overrightarrow{0} \\
0 \\
\stackrel{1}{0} \\
0\end{array}$ & 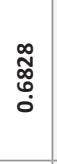 & 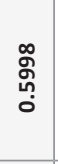 & 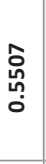 & $\begin{array}{c}\text { ্ָ } \\
\stackrel{g}{0} \\
0\end{array}$ & 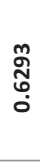 \\
\hline 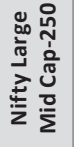 & $\Sigma$ & $\Sigma$ & $\Sigma$ & $\Sigma$ & $\Sigma$ & $\Sigma$ & $\Sigma$ & $\Sigma$ & $\begin{array}{l}\overleftarrow{\hat{N}} \\
\stackrel{-}{0} \\
0\end{array}$ & $\underset{0}{\stackrel{0}{0}}$ & 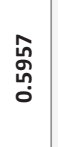 & $\begin{array}{l}\text { స్లి } \\
0 \\
0\end{array}$ & $\begin{array}{l}\stackrel{n}{\kappa} \\
\hat{b} \\
0\end{array}$ & $\begin{array}{l}n \\
0 \\
0 \\
0 \\
0\end{array}$ & 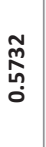 & 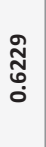 & $\begin{array}{l}\stackrel{\circ}{\circ} \\
\text { : } \\
0\end{array}$ \\
\hline 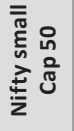 & $\Sigma$ & $\Sigma$ & $\Sigma$ & 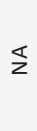 & $\Sigma$ & 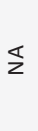 & $\Sigma$ & 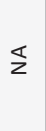 & 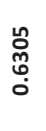 & $\begin{array}{l}\stackrel{2}{0} \\
\stackrel{0}{0} \\
0 \\
0\end{array}$ & \begin{tabular}{l}
$\infty$ \\
\multirow{2}{n}{} \\
\\
0
\end{tabular} & $\begin{array}{l}\overrightarrow{\hat{O}} \\
0 \\
0\end{array}$ & 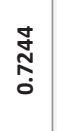 & $\begin{array}{l}\stackrel{0}{0} \\
\stackrel{0}{0} \\
0\end{array}$ & 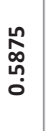 & $\begin{array}{l}\stackrel{N}{\tilde{N}} \\
\stackrel{0}{0} \\
0\end{array}$ & مे \\
\hline 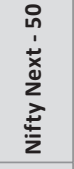 & $\begin{array}{l}\stackrel{0}{\circ} \\
\text { ஸ̂n } \\
0\end{array}$ & $\begin{array}{l}\tilde{N} \\
0 \\
0 \\
0\end{array}$ & 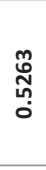 & 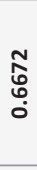 & $\begin{array}{l}\text { స్ } \\
\text { ర్ } \\
0\end{array}$ & 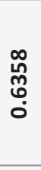 & 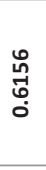 & 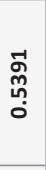 & 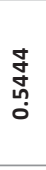 & $\begin{array}{l}\stackrel{0}{0} \\
\hat{6} \\
0 \\
0\end{array}$ & 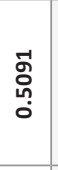 & 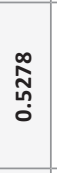 & 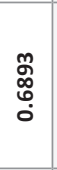 & 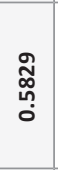 & 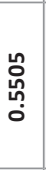 & $\begin{array}{l}\infty \\
0 \\
\stackrel{0}{0} \\
0\end{array}$ & 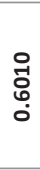 \\
\hline 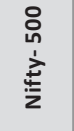 & $\Sigma$ & 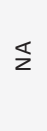 & 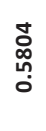 & 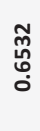 & 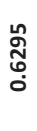 & 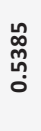 & $\begin{array}{l}\text { ô } \\
\text { to } \\
\stackrel{0}{0}\end{array}$ & $\begin{array}{l}\text { Sิ } \\
\text { ํํ } \\
\text { o. }\end{array}$ & 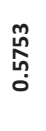 & $\begin{array}{l}0 \\
\stackrel{0}{0} \\
0\end{array}$ & $\underset{\substack{\hat{n} \\
0 \\
0}}{N}$ & 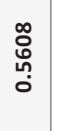 & 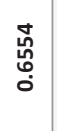 & $\begin{array}{l}\stackrel{\sim}{\tilde{W}} \\
0 \\
0\end{array}$ & $\begin{array}{l}\text { స̃ } \\
\text { ஸ̂? } \\
\stackrel{0}{0}\end{array}$ & 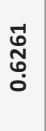 & $\begin{array}{l}\text { 苟 } \\
\text { f } \\
0\end{array}$ \\
\hline $\begin{array}{l}\stackrel{\text { D }}{1} \\
\frac{1}{2} \\
\frac{2}{2}\end{array}$ & 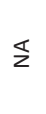 & $\Sigma$ & $\Sigma$ & $\Sigma$ & $\Sigma$ & $\Sigma$ & $\bar{z}$ & 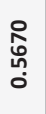 & 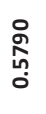 & $\begin{array}{l}\text { 员 } \\
00 \\
0\end{array}$ & 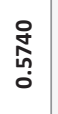 & $\begin{array}{c}\infty \\
\sum_{n}^{n} \\
0\end{array}$ & $\begin{array}{l}0 \\
0 \\
\vdots \\
0\end{array}$ & 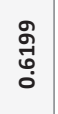 & 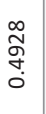 & 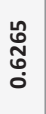 & $\begin{array}{l}\text { 员 } \\
\text { ĥ } \\
\text { ○े }\end{array}$ \\
\hline $\begin{array}{l}\stackrel{0}{0} \\
\stackrel{1}{3} \\
\frac{1}{2}\end{array}$ & $\Sigma$ & $\Sigma$ & $\Sigma$ & $\Sigma$ & $\Sigma$ & $\Sigma$ & 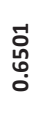 & 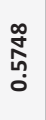 & $\begin{array}{l}n \\
\stackrel{0}{\infty} \\
\hat{\leftrightarrow} \\
0\end{array}$ & $\begin{array}{l}m \\
\stackrel{0}{o} \\
\stackrel{0}{0} \\
\stackrel{0}{0}\end{array}$ & 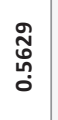 & $\begin{array}{l}m \\
g \\
\stackrel{n}{0} \\
0 \\
0\end{array}$ & $\begin{array}{l}\stackrel{\infty}{\mathbf{T}} \\
\stackrel{6}{0}\end{array}$ & 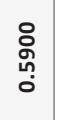 & 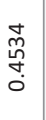 & $\begin{array}{l}\stackrel{n}{\tilde{N}} \\
\stackrel{0}{0}\end{array}$ & 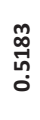 \\
\hline 号 & $\begin{array}{l}\hat{N} \\
\text { గn } \\
0\end{array}$ & $\begin{array}{l}\text { 它 } \\
\text { ผึ. } \\
0\end{array}$ & 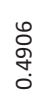 & $\begin{array}{l}\text { ồ } \\
\text { مُ } \\
\text { o }\end{array}$ & 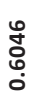 & 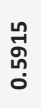 & $\begin{array}{l}\vec{N} \\
\qquad \\
0 \\
0\end{array}$ & 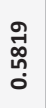 & 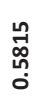 & $\begin{array}{l}\vec{d} \\
0 \\
0 \\
0\end{array}$ & $\underset{\substack{n \\
\\
0}}{0}$ & $\begin{array}{l}\text { ָิ } \\
\text { น̂n } \\
0\end{array}$ & $\begin{array}{l}\underset{\infty}{ت} \\
\stackrel{1}{0} \\
\stackrel{0}{0}\end{array}$ & 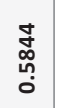 & $\begin{array}{c}\stackrel{\infty}{\underset{f}{f}} \\
\stackrel{0}{0}\end{array}$ & $\begin{array}{l}0 \\
0 \\
0 \\
0 \\
0\end{array}$ & $\begin{array}{l}\text { L } \\
\text { ğ } \\
\text { o. }\end{array}$ \\
\hline 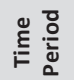 & ડ్తి & $\stackrel{\infty}{2}$ & જે & ¿ั. & ठ̊. & రิ & ֻి & ఫั & 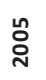 & ๕ั & ڤે̀े & $\stackrel{\text { ڤ̊ }}{\text { ¿े }}$ & ڤ્ & 오 & নั & ปั & $\stackrel{m}{\stackrel{n}{N}}$ \\
\hline
\end{tabular}




\begin{tabular}{|c|c|c|c|c|c|c|}
\hline 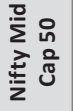 & $\begin{array}{l}n \\
0 \\
0 \\
0 \\
0\end{array}$ & $\begin{array}{l}\stackrel{\mathbb{N}}{\tilde{J}} \\
\stackrel{0}{0}\end{array}$ & $\begin{array}{l}\text { N్ } \\
\text { Co } \\
\stackrel{0}{0}\end{array}$ & 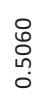 & 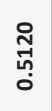 & \begin{tabular}{l}
$\mathbb{Z}$ \\
\multirow{J}{0}{} \\
0
\end{tabular} \\
\hline 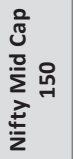 & 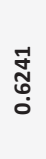 & $\begin{array}{l}\Re 䶹 \text { } \\
\stackrel{+}{+} \\
\stackrel{0}{0}\end{array}$ & $\begin{array}{l}\stackrel{9}{7} \\
\text { గ్ } \\
\stackrel{0}{0}\end{array}$ & 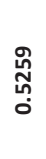 & $\begin{array}{l}\hat{o} \\
\hat{n} \\
0 \\
0\end{array}$ & $\begin{array}{l}\text { L } \\
\infty \\
0 \\
0 \\
0 \\
0\end{array}$ \\
\hline 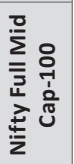 & 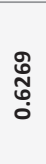 & $\begin{array}{l}0 \\
0 \\
o \\
+ \\
0\end{array}$ & $\begin{array}{l}\tilde{o} \\
\tilde{ల} \\
\stackrel{0}{0}\end{array}$ & 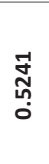 & 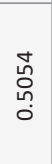 & $\begin{array}{l}\hat{N} \\
\text { ơ } \\
\text { ல }\end{array}$ \\
\hline 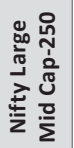 & 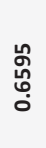 & 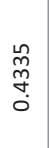 & $\begin{array}{l}\text { श्रे } \\
\text { फ़ } \\
\text { ¿ }\end{array}$ & 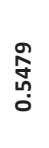 & $\begin{array}{l}\hat{b} \\
0 \\
0 \\
0\end{array}$ & $\begin{array}{c}\underset{N}{N} \\
\text { ñ } \\
0\end{array}$ \\
\hline 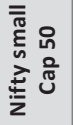 & 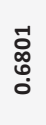 & $\begin{array}{l}\underset{g}{g} \\
\underset{f}{0} \\
\stackrel{0}{*}\end{array}$ & $\begin{array}{l}\hat{0} \\
\hat{6} \\
0\end{array}$ & 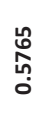 & $\begin{array}{l}\text { นू⿱ } \\
\text { గ్ } \\
\text { ○े }\end{array}$ & \begin{tabular}{l}
0 \\
\multirow{\circ}{0}{} \\
0 \\
0
\end{tabular} \\
\hline 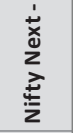 & $\begin{array}{l}\text { ָू్ } \\
\text { ஸ̂. } \\
0\end{array}$ & $\begin{array}{l}\stackrel{0}{0} \\
\tilde{y} \\
\stackrel{0}{0}\end{array}$ & $\begin{array}{l}\text { 유 } \\
\text { ب̂ } \\
\stackrel{0}{0}\end{array}$ & $\begin{array}{l}\stackrel{\sim}{7} \\
\stackrel{m}{+} \\
\stackrel{0}{0}\end{array}$ & 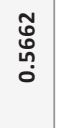 & 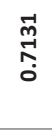 \\
\hline 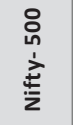 & 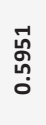 & $\begin{array}{l}\stackrel{\hat{~}}{\sim} \\
\stackrel{\circ}{0}\end{array}$ & 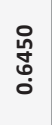 & 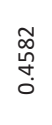 & 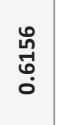 & $\begin{array}{l}\stackrel{J}{J} \\
\stackrel{G}{0} \\
0\end{array}$ \\
\hline 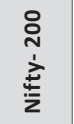 & $\begin{array}{l}\text { : } \\
\text { \& } \\
\text { ஸ̂. } \\
0\end{array}$ & $\begin{array}{l}\stackrel{\infty}{\sim} \\
\stackrel{\sim}{0}\end{array}$ & 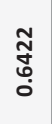 & \begin{tabular}{l}
$\hat{n}$ \\
\multirow{5}{*}{} \\
$\dot{0}$
\end{tabular} & 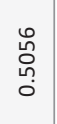 & $\begin{array}{l}\stackrel{0}{N} \\
60 \\
0\end{array}$ \\
\hline $\begin{array}{l}\stackrel{8}{\circ} \\
\stackrel{+}{1} \\
\stackrel{1}{2}\end{array}$ & 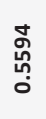 & $\begin{array}{l}\underset{\widetilde{\sim}}{\sim} \\
\stackrel{\sim}{0}\end{array}$ & $\begin{array}{l}\stackrel{m}{7} \\
\stackrel{G}{6} \\
\stackrel{0}{0}\end{array}$ & $\begin{array}{l}\stackrel{7}{7} \\
\stackrel{7}{+} \\
0\end{array}$ & $\begin{array}{l}\stackrel{m}{\tilde{N}} \\
\stackrel{0}{0}\end{array}$ & $\begin{array}{l}\stackrel{0}{N} \\
60 \\
0\end{array}$ \\
\hline in & 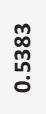 & $\begin{array}{l}\stackrel{\infty}{\tilde{m}} \\
\stackrel{\sigma}{0}\end{array}$ & $\begin{array}{l}\overrightarrow{7} \\
\stackrel{\oplus}{0} \\
0 \\
0\end{array}$ & 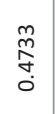 & $\begin{array}{l}0 \\
0 \\
0 \\
\\
0\end{array}$ & 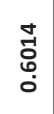 \\
\hline 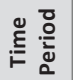 & ন্ & مै & 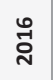 & $\hat{\tilde{N}}$ & 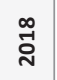 & స్ \\
\hline
\end{tabular}


Table 4 presents estimated long memory during year wise analysis of Nifty-50, Nifty-100, Nifty- 200, Nifty-500, Nifty Next -50, Nifty small Cap 50, Nifty Large Mid Cap-250, Nifty Full Mid Cap-100, Nifty Mid Cap 150 and Nifty Mid Cap 50 using Rescaled Range Statistics. The estimated H coefficient suggests that from 1997 to 2019 almost all indices in each year show significant persistence behavior with the exception of Nifty full Mid Cap-100 in year 2007, Nifty- 50, Nifty-100, Nifty-200, and Nifty-500 in year 2011, Nifty-50, Nifty-200, Nifty-500, Nifty Next-50 and Nifty Mid Cap -50 in year 2017, Nifty-50, Nifty-500, Nifty Large Mid Cap-250, and Nifty Full Mid Cap-100 in year 2018 and Nifty Full Mid Cap-100 in year 2019. However, 2015 was the year when all indices exhibit anti-persistence behavior. It is pertinent to note that year 2014 observed significant political change from the Congress Lead Government to the NDA lead Government, which brought significant changes in the political, regulatory and governance environment in India. Moreover, on November 8, 2016 a major announcement was made by Prime Minister Mr. Narender Modi regarding demonetization of Rs. 1,000 and Rs. 500 currency notes, which amounted to nearly $86 \%$ of the total currency in circulation. This announcement brought a revolution in the Indian economy and there was major shift in the mode of transactions from cash to digital modes of transactions and change in the outlook of Indian capital market for foreign investors ${ }^{1}$ (Booth \& Tse, 1995), (Cheung \& Lai, 1995) and (Cont, 2005).

Table 5 presents the each year analysis of long memory of all indices in liquidity series. Results indicate that Nifty-50 showing significant long memory in the years 1998, 1999, 2001, 2009, 2010, 2011, 2014, 2016, 2017, 2018, and 2019. Furthermore, Nifty 100 displays persistence behavior in 2009, 2010, 2014, 2015, 2016, 2017, and 2018. Nifty-200 shows long memory in 2011, 2014, and 2018. This indicates possibility of predictable component of past liquidity (Huang \& Yang, 1999) and (Hiremath \& Kamaiah, 2010). Nifty-500 exhibits persistence behavior in 2001, 2009 and 2010and Nifty Next -50 advocate's long memory in 1998, 1999, 2000, 2001, 2009, 2014, 2015, 2016, and 2017. Moreover, Nifty small Cap 50, Nifty full Mid Cap-100 and Nifty Mid Cap 150 shows serial correlation behavior only in the year 2019 .

1 “Theriseofsmall-towninvestorsinIndianequitymarket" (https://economictimes. indiatimes.com/markets/stocks/news/the-rise-of-small-town-investors-in- indianequity-markets/articleshow/71270423.cms?from=mdr). 


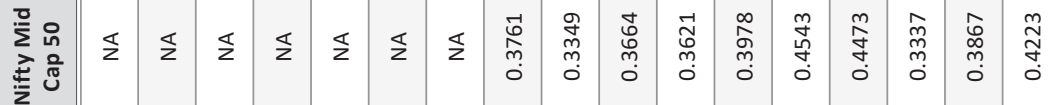

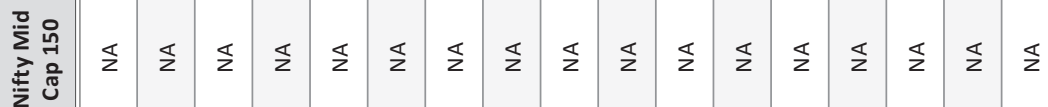

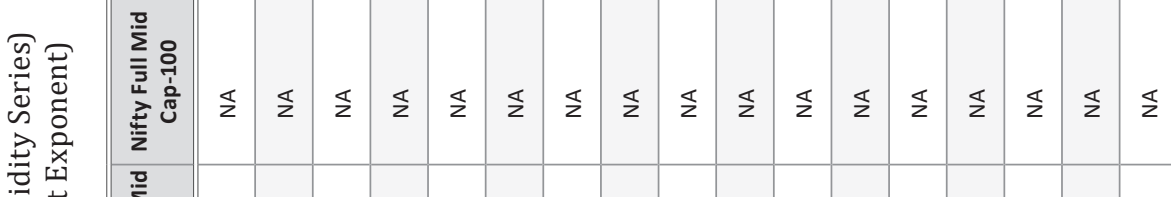
莺

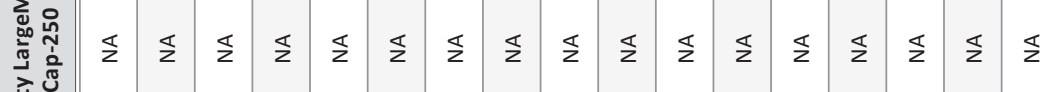
岂

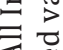

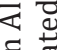

政

ำ

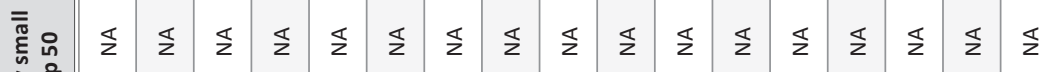
菜

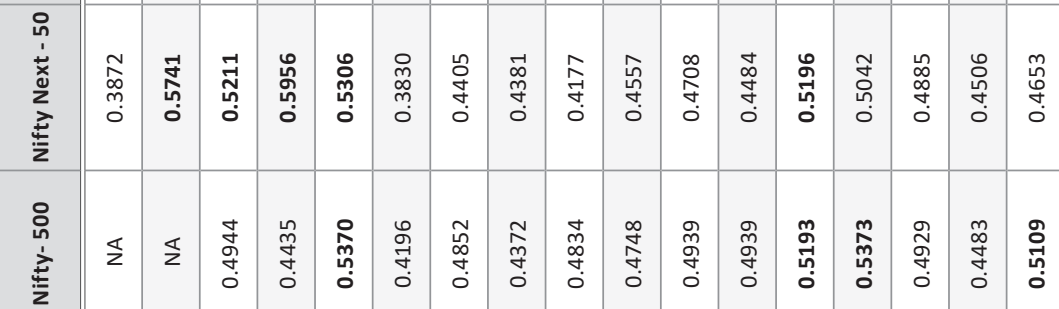

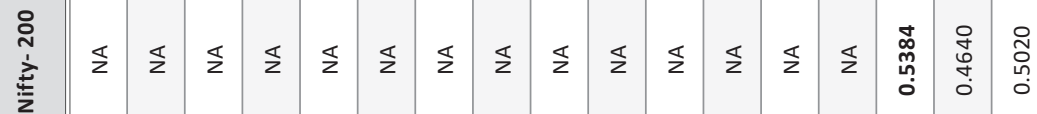

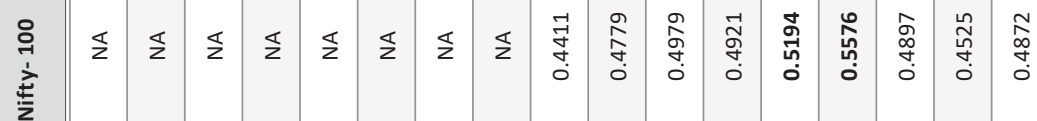

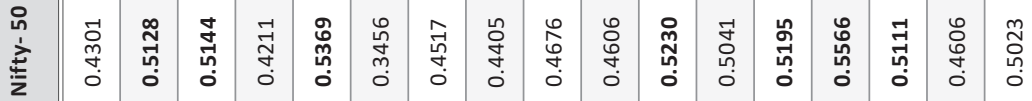

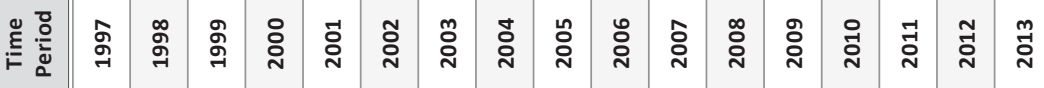




\begin{tabular}{|c|c|c|c|c|c|c|}
\hline 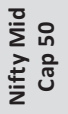 & 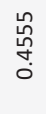 & $\begin{array}{l}\stackrel{\sim}{\sim} \\
\underset{7}{+} \\
\stackrel{0}{0}\end{array}$ & 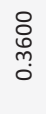 & $\begin{array}{l}\text { İ } \\
\text { o } \\
0 \\
0\end{array}$ & $\underset{\substack{N \\
\sim \\
0}}{\stackrel{N}{0}}$ & 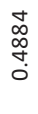 \\
\hline 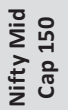 & $\Sigma$ & $\Sigma$ & $\begin{array}{l}\text { O্+ } \\
\stackrel{m}{m} \\
0\end{array}$ & \begin{tabular}{l}
-1 \\
\multirow{0}{0}{} \\
+ \\
0 \\
0
\end{tabular} & $\underset{\substack{m \\
\tilde{d}}}{\stackrel{m}{0}}$ & 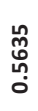 \\
\hline 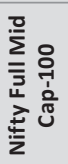 & $\Sigma$ & $\Sigma$ & $\begin{array}{c}\text { ণ } \\
\text { ஸे } \\
0\end{array}$ & $\begin{array}{l}0 \\
0 \\
0 \\
0 \\
0\end{array}$ & 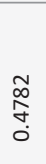 & $\begin{array}{l}\hat{\circ} \\
\text { గ̂t } \\
0\end{array}$ \\
\hline 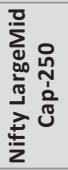 & $\Sigma$ & $\Sigma$ & $\Sigma$ & 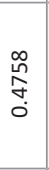 & 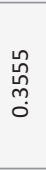 & 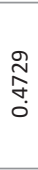 \\
\hline 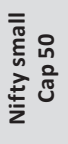 & $\Sigma$ & $\Sigma$ & $\begin{array}{l}m \\
\infty \\
\stackrel{n}{*} \\
\stackrel{0}{0}\end{array}$ & $\begin{array}{l}\text { M } \\
\stackrel{0}{f} \\
0\end{array}$ & $\underset{\substack{n \\
m}}{0}$ & $\begin{array}{l}\mathscr{0} \\
0 \\
0 \\
0\end{array}$ \\
\hline 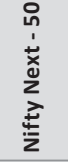 & 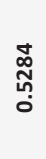 & 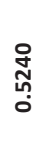 & $\begin{array}{l}\stackrel{\text { n}}{7} \\
\text { กุ } \\
0\end{array}$ & 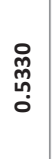 & $\begin{array}{c}\underset{J}{J} \\
\stackrel{m}{0} \\
\stackrel{0}{*}\end{array}$ & $\begin{array}{l}n \\
0 \\
0 \\
0 \\
0\end{array}$ \\
\hline 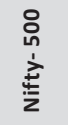 & $\begin{array}{l}\text { शे } \\
\text { Оे } \\
\text { ஸे } \\
0\end{array}$ & $\begin{array}{l}\stackrel{9}{+} \\
\stackrel{+}{+} \\
\stackrel{0}{0}\end{array}$ & $\begin{array}{l}\stackrel{n}{n} \\
\stackrel{n}{n} \\
0\end{array}$ & 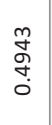 & $\begin{array}{l}\text { 엄 } \\
\text { ơ } \\
\text { ○े }\end{array}$ & $\begin{array}{l}\text { } \\
\text { oे } \\
\text { fे } \\
0\end{array}$ \\
\hline 定 & 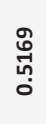 & $\begin{array}{l}\text { n } \\
\infty \\
\stackrel{+}{+} \\
\stackrel{0}{0}\end{array}$ & $\begin{array}{l}\circ \\
\stackrel{0}{0} \\
\leftrightarrow \\
0\end{array}$ & $\begin{array}{l}0 \\
\exists \\
\text { กี่ } \\
0\end{array}$ & 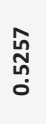 & $\begin{array}{l}\vec{\Xi} \\
\text { ఫे } \\
0\end{array}$ \\
\hline 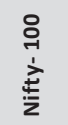 & 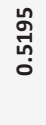 & $\begin{array}{l}\hat{m} \\
\text { గñ } \\
0\end{array}$ & 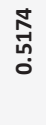 & $\begin{array}{l}\text { N̂̃ } \\
\text { గุ. }\end{array}$ & 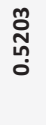 & $\begin{array}{l}\text { İ } \\
\text { ఫे } \\
\text { Oे }\end{array}$ \\
\hline 온 & 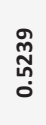 & \begin{tabular}{l}
$\underset{f}{J}$ \\
\multirow{f}{*}{} \\
0
\end{tabular} & \begin{tabular}{l}
$\mathscr{0}$ \\
\multirow{1}{n}{} \\
0
\end{tabular} & 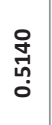 & 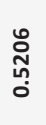 & 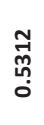 \\
\hline 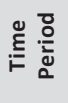 & ন্̀ & ڤั & ڤ̊: & ثิ̀ & $\stackrel{\infty}{\stackrel{\sim}{\sim}}$ & 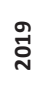 \\
\hline
\end{tabular}


Furthermore, Nifty large Mid Cap-250 and Nifty Mid Cap 50 did not show the significant long memory, which may arise due to micro and macro factors i.e. changes in interest rates, inflation or deflation and happenings in global markets (Kang \& Yoon, 2007), (Asian crisis, IT bubbles, Indo-Pak political crisis and Real estate bubbles) are counted as the significant ones (Gurgul \& Wojtowicz, 2006), (Christodoulou-Volos \& Siokis, 2006), and (Pandy, April14, 2018)².

\section{DISCUSSION AND IMPLECATION}

Result of present study focuses on measurement of the portfolio management for retail investors and first time investors who are going to invest their money into the market, where they could consider the effect of all micro and macro factors on their investment strategy. Moreover, findings would be beneficial for the academician, practitioners, policy makers, portfolio manager and investors, whose decision depend upon the market predictability, therefore, present study provide insights for better understanding and is useful for forecasting to take financial decision. Findings are consistent with (Hiremath \& Kamaiah, 2011), (Sadique \& Silvapulle, 2001), (Henry, 2002), (Cavalcante \& Assaf, 2004; Booth \& Tse, 1995; Turkyilmaz \& Balibey, 2014).

\section{CONCLUSION}

The present study is an attempt to examine the presence of long memory in broad stock Indices at National Stock Exchange of India. Using daily log returns and liquidity of Nifty-50, Nifty-100, Nifty-200, Nifty-500, Nifty Next -50, Nifty Small Cap 50, Nifty Large Mid Cap-250, Nifty Full Mid Cap-100, Nifty Mid Cap-150 and Nifty Mid Cap-50 index in India Hurst Exponent in Rescaled Range Analysis has been estimated. The results of the study confirm presence of long memory in returns of all indices during full sample period. However, in case of liquidity series, it shows persistent nature in Nifty Mid Cap 50 only. Moreover, for year-wise analysis of long memory has been estimated for all indices. Findings suggest significant long memory in returns series across all indices except

2 See Source: https://www.financialexpress.com/market/three-key-economicfactors-which-affect-sensex-nifty/1132894/ (Pandy, April ,14, 2018, "Three key economic factors which affect Sensex, Nifty" Financial Express). 
for the years 2015 and 2017 whereas, in case of liquidity, all indices show antipersistence behavior. An important observation from the year-wise analysis is that nonetheless various regulatory, technological, structural changes have taken place in the Indian equity market during last two decades but the price discovery in Indian equity market is yet not efficient (Mukherjee, Sen \& Sarkar, 2011), (Verma, 2008), (Hassler \& Wolters, 1995), (Baillie \& Morana, 2009), (Caporale \& Gil-Alana, 2008) and (Limam, 2003).

\section{REFERENCES}

Aydogan, K., \& Booth, G. G. (1988). Are there long cycles in common stock returns? Southern Economic Journal, 141-149.

Badhani, K. N. (2006). Empirical Regularities in the Intra-Week Trading Patterns of Foreign Institutional Investors in India. Working Paper SSRN, http://dx.doi. org/10.2139/ssrn.900501.

Badhani, K. N. (2008) Long memory in stock returns and volatility in India: A nonparametric analysis. ICFAI Journal of Applied Finance, 14(12), 34-53.

Badhani, K. N. (2012). Does Nifty have a Long Memory? Semi-parametric Estimation of Fractional Integration in Returns and Volatility. Decision, 39(3),86-100.

Baillie, R. T. (1996). Long memory processes and fractional integration in econometrics. Journal of econometrics, 73(1), 5-59.

Baillie, R. T., \& Morana, C. (2009). Modelling long memory and structural breaks in conditional variances: An adaptive FIGARCH approach. Journal of Economic Dynamics and Control, 33(8), 1577-1592.

Bala, A., \& Gupta, K. (2018). Testing the long memory feature in Indian equity market. Journal of Research in Management, 10(2), 24-33.

Balcilar, M., Ozdemir, Z. A., \& Cakan, E. (2015). Structural breaks, long memory or unit roots in stock prices: Evidence from emerging markets. International Econometric Review (IER), 7(1), 13-33.

Bariviera, A. F. (2011). The influence of liquidity on informational efficiency: The case of the Thai Stock Market. Physica A: Statistical Mechanics and its Applications, 390(23-24), 4426-4432.

Barkoulas, J. T., \& Baum, C. F. (1996). Long-term dependence in stock returns. Economics Letters, 53(3), 253-259.

Barkoulas, J. T., Baum, C. F., \& Travlos, N. (2000). Long memory in the Greek stock market. Applied Financial Economics, 10(2), 177-184.

Baum, C. F., Barkoulas, J. T., \& Caglayan, M. (1999). Long memory or structural breaks: Can either explain nonstationary real exchange rates under the current float? Journal of International Financial Markets, Institutions and Money, 9(4), 359-376.

Bhattacharya, S. N., \& Bhattacharya, M. (2012). Long memory in stock returns: A study of emerging markets. Iranian Journal of Management Studies, 5(5), 67-88. 
Bhattacharya, S. N., \& Bhattacharya, M. (2013). Long memory in return structures from developed markets. Management Letters, 13(2), 127-143. http://dx.doi.org/ 10.5295/cdg.110312sb.

Bhattacharya, S. N., Sengupta, P., Bhattacharya, M., \& Roychoudhury, B. (2016). Multidimensional liquidity: Evidences from Indian stock market. Applied Finance Letters, 5(2). http://dx.doi.org/10.24135/afl.v5i2.47.

Booth, G. G., \& Tse, Y. (1995). Long memory in interest rate futures markets: A fractional cointegration analysis. Journal of Futures Markets, 15(5), 573-584.

Cajueiro, D. O., \& Tabak, B. M. (2005). Possible causes of long-range dependence in the Brazilian stock market. Physica A: Statistical Mechanics and its Applications, 345(3), $635-645$.

Cajueiro, D. O., \& Tabak, B. M. (2008). Testing for long-range dependence in world stock markets. Chaos, Solitons \& Fractals, 37(3), 918-927.

Caporale, G. M., \& Gil-Alana, L. A. (2008). Long memory and structural breaks in the Spanish stock market index. Open Operational Research Journal, 2, 13-17.

Cavalcante, J., \& Assaf, A. (2004). Long range dependence in the returns and volatility of the Brazilian stock market. European review of Economics and Finance, 3(5), 22.

Cevik, P., \& Emec, H. (2013). Long memory properties in return and volatility: An application of the impact of arab spring in turkey financial market. Current Research Journal of Social Sciences, 5(2), 60-66.

Chen, J. H., \& Diaz, J. F. (2013). Long memory and shifts in the returns of green and nongreen Exchange- Traded Funds (ETFs). International Journal of Humanities and Social Science Invention, 2(10), 29-32.

Chen, J. H., \& Huang, Y. F. (2014). Long memory and structural breaks in modelling the volatility dynamics of VIX-ETFs. International Journal of Business, Economics and Law, 4(1), 54-63.

Cheung, Y. W., \& Lai, K. S. (1995). A search for long memory in international stock market returns. Journal of International Money and Finance, 14(4), 597-615.

Chow, K. V., Denning, K. C., Ferris, S., \& Noronha, G. (1995). Long-term and short-term price memory in the stock market. Economics Letters, 49(3), 287-293.

Christodoulou-Volos, C., \& Siokis, F. M. (2006). Long range dependence in stock market returns. Applied Financial Economics, 16(18), 1331-1338.

Chung, H., Lin, W. T., \& Wu, S. (2000). An analysis of long memory in volatility for Asian stock markets. Review of Pacific Basin Financial Markets and Policies, 3(3), 309-330.

Cont, R. (2005). Long range dependence in financial markets. In Fractals in Engineering, pp. 159-179. London: Springer.

Danilenko, S. (2009). Long-term memory effect in stock prices analysis. Economics and management, 14, 151-155.

Fama, E. F. (1970). Efficient capital markets: A review of theory and empirical work. The journal of Finance, 25(2), 383-417.

Fama, E. F. (1991). Efficient capital markets: II. The Journal of Finance, 46(5), 1575-1617.

Garvey, J., \& Gallagher, L. (2009). Fractional Integration in Equity Volatility. 
Gayathri, M., Murugesan, S., \& Gayathri, J. (2012). Persistence and long range dependence in Indian stock market returns. International Journal of Management and Business Studies, 2(4), 72-77.

Goudarzi, H., \& Ramanarayanan, C. S. (2011). Modeling asymmetric volatility in the Indian stock market. International Journal of Business and Management, 6(3), 221.

Greene, M. T., \& Fielitz, B. D. (1977). Long-term dependence in common stock returns. Journal of Financial Economics, 4(3), 339-349.

Gurgul, H., \& Wojtowicz, T. (2006). Long memory on the German stock exchange. Czech Journal of Economics and Finance (Finance a uver), 56(9-10), 447-468.

Hassler, U., \& Wolters, J. (1995). Long memory in inflation rates: International evidence. Journal of Business and Economic Statistics, 13(1), 37-45.

Henry, O. T. (2002). Long memory in stock returns: some international evidence. Applied financial economics, 12(10), 725-729.

Hiremath, G. S., \& Kamaiah, B. (2010). Long memory in stock market volatility: Indian evidences. ArthaVijnana, 52(4), 332-345.

Hiremath, G. S., \& Kamaiah, B. (2011). Testing long memory in stock returns of emerging markets: Some further evidence. Economics, Management, and Financial Markets, 6(3), 136-147.

Huang, B. N., \& Yang, C. W. (1999). An examination of long-term memory using the intraday stock returns. (Technical Report 99-03). Clarion: Clarion University of Pennsylvania.

Hull, M., \& McGroarty, F. (2014). Do emerging markets become more efficient as they develop? Long memory persistence in equity indices. Emerging Markets Review, 18, 45-61.

Hurst, H. E. (1951) The long-term storage capacity of reservoir. Transactions of the American Society of Civil Engineers, 116(1), 770-799.

Jayasuriya, S. A. (2009). A Sub Period Analysis of Long Memory in Stock Return Volatility. In 9th Global Conference on Business and Finance.

Kang, S. H., \& Yoon, S. M. (2007). Long memory properties in return and volatility: Evidence from the Korean stock market. Physica A: Statistical Mechanics and its Applications, 385(2), 591-600.

Kilic, R. (2004). On the long memory properties of emerging capital markets: evidence from Istanbul stock exchange. Applied Financial Economics, 14(13), 915-922.

Krishnan, R., \& Mishra, V. (2013). Intraday liquidity patterns in Indian stock market. Journal of Asian Economics, 28, 99-114.

Kumar, A. (2004). Long memory in stock trading volume: Evidence from Indian stock market. Working Paper SSRN. http://dx.doi.org/10.2139/ssrn.557681.

Kumar, D. (2014). Long-range dependence in Indian stock market: A study of Indian sectoral indices. International Journal of Emerging Markets, 9(4), 505-519.

Kumar, G., \& Misra, A. K. (2018). Commonality in liquidity: Evidence from India's national stock exchange. Journal of Asian Economics, 59, 1-15. 
Lillo, F., \& Farmer, J. D. (2004). The long memory of the efficient market. Studies in nonlinear dynamics \& econometrics, 8(3).

Limam, I. (2003). Is long memory a property of thin stock markets? International Evidence usingArab countries. Review of Middle East Economics and Finance, 1(3), 251-266.

Lo, A. (1991). Long term memory in stock market prices. Econometrica, 59(5), 1279$-1313$.

Ma, C., Li, H., Zou, L., \& Wu, Z. (2006). Long-term memory in emerging markets: evidence from the Chinese stock market. International Journal of Information Technology \& Decision Making, 5(03), 495-501.

Mahalingam, G., \& Selvam, M. (2014). Stock market reaction during the global financial crisis in India: fractal analysis. Asia-Pacific Journal of Management Research and Innovation, 10(4), 403-412.

Mandelbrot, B. B. (1971). When can price be arbitraged efficiently? A limit to the validity of the random walk and martingale models. The Review of Economics and Statistics, 225-236.

Mandelbrot, B. B. (1997). The variation of certain speculative prices. In Fractals and scaling in finance. New York: Springer. https://doi.org/10.1007/978-1-4757-27630_14.

Mandelbrot, B. (1963). The variation of certain speculative prices. Journal of Business, 36, 394-419.

Mukherjee, I., Sen, C., \& Sarkar, A. (2011). Long memory in stock returns: Insights from the Indian market. The International Journal of Applied Economics and Finance, 5(1), 62-74.

Nath, G. C., \& Reddy, Y. V. (2002). Long memory in rupee-dollar exchange rate: An empirical study. In Capital Market Conference, December 2002.

Onour, I. A. (2010). North Africa stock markets: Analysis of long memory and persistence of shocks. International Journal of Monetary Economics and Finance, 3(2), 101$-111$.

Ozun, A., \& Cifter, A. (2007). Modeling Long-Term Memory Effect in Stock Prices: A Comparative Analysis with GPH Test and Daubechies Wavelets. Studies in Economics and Finance, 25(1), 38-48. http://dx.doi.org/10.1108/10867370810857559.

Peters, E.E. (1994), Fractal Market Analysis, New York: John Wiley nad Sons, Inc.

Peters, E.E. (1996), Chaos and Order in the Capital Markets. 2nd Edition, John Wiley and Sons, Inc.

Sadique, S., \& Silvapulle, P. (2001). Long- term memory in stock market returns: International evidence. International Journal of Finance and Economics, 6(1), 59-67.

Soofi, A. S., Wang, S., \& Zhang, Y. (2006). Testing for long memory in the Asian foreign exchange rates. Journal of Systems Science and Complexity, 19(2), 182-190.

Souza, S. R., Tabak, B. M., \& Cajueiro, D. O. (2008). Long-range dependence in exchange rates: The case of the European monetary system. International Journal of Theoretical and Applied Finance, 11(2), 199-223. 
Turkyilmaz, S., \& Balibey, M. (2014). Long memory behavior in the returns of Pakistan stock market: ARFIMA-FIGARCH models. International Journal of Economics and Financial Issues, 4(2), 400-441.

Verma, A. (2008). Long memory of the Indian stock market. The IUP Journal of Financial Economics, 6(3), 74-83. 